\title{
Revealing Genetic Variability and Trait Association Studies in Landraces of Rice (Oryza sativa L.) Under Controlled and Drought Conditions
}

\author{
H.R. Uday Kumar ${ }^{1 *}$, S. Gangaprasad ${ }^{2}$, P. Raghavendra ${ }^{2}$ and B.M. Dushyantha Kumar ${ }^{2}$ \\ ${ }^{1}$ Departmentof Genetics and Plant Breeding, University of Agricultural Sciences (UAS), \\ Bengaluru, Karnataka, India \\ ${ }^{2}$ Departmentof Genetics and Plant Breeding, University of Agricultural and Horticultural \\ Sciences (UAHS), Shimoga, Karnataka, India \\ *Corresponding author
}

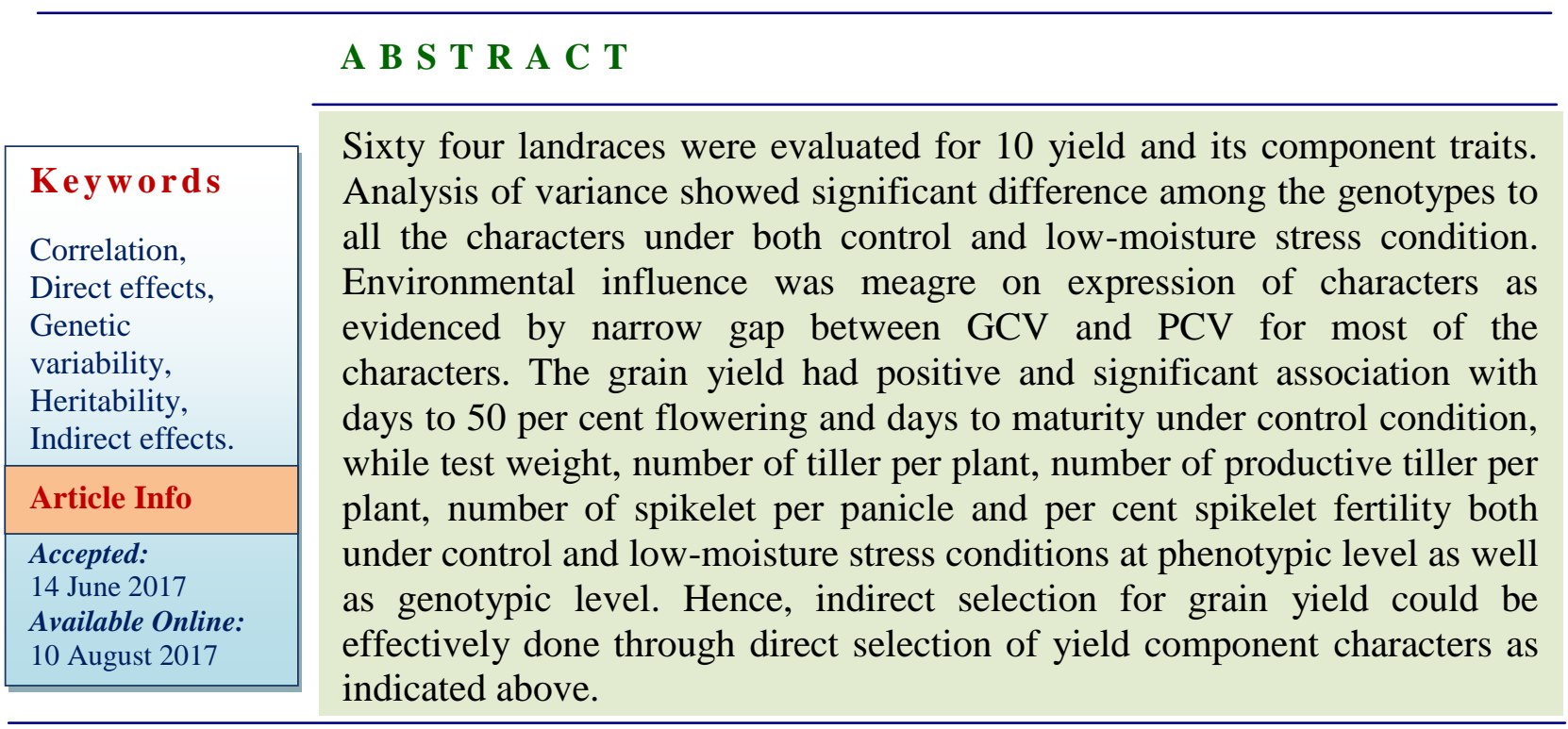

\section{Introduction}

Rice (Oryza sativa L.) is a highly domesticated crop, and domestication processes are reported to be accompanied by genetic erosion, which causes a reduction in genetic diversity among traditional varieties and gradual loss of landraces from the fields (Brush, 2000). An exhaustive survey of genetic variability and detailed understanding of genetic makeup of the crop is an important prerequisite for initiating crop improvement programme. The variability observed in any population could be due to the genetic and environment factors and also due to interaction between these factors. The genetic and environmental components of variation were discussed by Johannsen (1909) who attributed the variation in a segregating population to heritable and non-heritable factors, while the variation within pure lines was attributed to environmental factors. The heritable variations could be further divided into additive and non-additive components. The heritable variations are also divided into dominant and inter-allelic interaction 
(Falconer, 1981). The broad sense heritability is the ratio of genotypic variance to the total variance in non-segregating population (Lush, 1945 and Hanson et al., 1956). The genotypic variance includes non-additive components which are not transmitted to next generation. Hence high heritability coupled with high genetic advance was reported to be more useful in practicing selection in a population (Johnson et al., 1955). It is essential to know the degree of mutual association prevailing between yield and its component traits, which forms the basis for selecting desirable genotypes. Correlation studies among these will provide an opportunity to quantify the magnitude and direction of their association along with other direct and indirect components. A study of Correlation and path analysis will thus help in identifying suitable selection criteria for improving yield. Hence the present study was undertaken to assess the variability, correlation and path coefficients in selected landraces of rice.

\section{Materials and Methods}

The material for the present study consisted of 64 traditional rice genotypes drawn from the rice germplasm maintained at Organic Farm Research Centre, Zonal Agricultural Research Station, Navile, Shimoga. The investigation was carried out at Organic Farm Research Centre, Navile, Shimoga and University of Agricultural Sciences (UAS), Bangalore. The rice genotypes included in the study were of diverse nature mentioned in Appendix I. The crop for the present investigation was raised in field as well as PVC pipes during Rabi season 2012-2013. The experiment was laid out in $8 \times 8$ Simple Lattice Design. For this design all 64 traditional genotypes were arranged in 8 columns and 8 rows with two replications under control and low-moisture stress condition. Low-moisture stress was imposed by withholding the irrigations from 30 days after transplanting to harvesting and the plants were given lifesaving irrigations only when they showed wilting symptoms, whereas in control plot regular irrigations were given without creating any stress. The spacing maintained was $30 \mathrm{~cm}$ between rows and $20 \mathrm{~cm}$ between plants within a row and other recommended agronomic practices were adapted to raise the crop. Five plants were selected at random from each entry in the replications for recording the observation on 10 metric characters. The averages of observations recorded on these five plants were considered for statistical analysis. The characters on which observations were recorded as per Standard evaluation system for rice (SES 1996) are Days to 50 per cent flowering, Days to maturity, Plant height (cm), 1000-grain weight (g), Number of tillers per plant, Number of productive tillers per plant (Number of panicles), Number of spikelet per panicle, Per cent spikelet fertility, Grain yield per plant $(\mathrm{g})$, The data of mean value for all the characters were analyzed for their variance following simple lattice design outlined by Cochran and Cox (1957). Correlation coefficients were computed as suggested by Snedecor and Cochran, (1965) and direct and indirect effects of yield components on yield were computed through path coefficient analysis as suggested by Dewey and Lu (1959) using WINDOSTAT software.

\section{Results and Discussion}

\section{Variability and genetic parameter}

The analysis of variance revealed highly significant difference among the germplasm accessions for all the traits indicating a large amount of variability was present in the set of material for effective selection (Table 1). Earlier workers like Abarshahr et al., (2011) and Osman et al., (2012) have reported the significant differences among the genotypes for all the characters they considered. The 
estimates of mean and range for all 10 quantitative traits were indicated in table 2 . The magnitude of phenotypic and genotypic coefficient of variations (PCV and GCV) was higher for yield and yield attributing traits but the difference is very less indicates the presence of environmental influence to some degree in the phenotypic expression of the traits (Table 2). The phenotypic and genotypic coefficient of variations were found very high for Grain yield/plant, followed by number of productive tillers per plant in both controlled and drought conditions. These results are in conformity with the findings of earlier workers Nagabhushan (2003) and Pandey et al., (2012). Whereas lowest PCV and GCV were recorded for Days to maturity and Days to fifty percent flowering in both controlled and drought condition. Madhusudhan (2001) also reported the similar results. The occurrence of low estimates of PCV and GCV indicated that selection directly based on these traits would not be much rewarding. The estimates of PCV were slightly higher than corresponding GCV for all the characters under study. Remabai et al., (1992) reported higher phenotypic coefficient of variation for all yield attributing parameters than genotypic coefficient of variation. Das et al., (2001) and Pandey et al., (2012) also noticed higher PCV than their respective GCV for all the traits studied by them.

The high estimates of heritability in broad sense with high genetic advance in per cent mean were observed for plant height, test weight, number of spikelet per panicle, per cent spikelet fertility and grain yield in both control and low-moisture stress condition, while for number of tillers per plant in lowmoisture stress and for number of productive tillers per plant in control condition (Table 2).

Similar results were obtained for plant height, test weight, number of spikelets per panicle and grain yield by Das et al., (2001) and Kuldeep et al., (2004) reported high heritability coupled with high genetic advance for yield components, Mall et al., (2005) reported high heritability and genetic advance for plant height, number of tillers per plant and number of spikelet per panicle.

\section{Correlation}

The result pertaining to correlation at phenotypic and genotypic levels between grain yield and other related characters are presented first fallowed by correlation among the characters under both control and lowmoisture stress conditions. Significant and positive association at phenotypic and genotypic level under control condition was found between grain yield with days to $50 \%$ flowering $(\mathrm{r}=0.255 ; 0.281)$, days to maturity (0.334; 0.384), 1000-grain weight (g) (0.399; $0.470)$, number of tillers per plant $(0.359$; 0.343 ), number of productive tillers per plant $(0.515 ; 0.536)$, number of spikelet per panicle $(0.394 ; 0.403)$ and per cent spikelet fertility $(0.441 ; 0.432)$ respectively (Tables 3 and 4$)$. Other yield components, viz., plant height, and panicle length showed non-significant positive association, Under low-moisture stress condition significant and positive association at phenotypic and genotypic level was found between grain yield with 1000grain weight $(\mathrm{g})(0.250 ; 0.261)$, number of tillers per plant $(0.377 ; 0.371)$, number of productive tillers per plant $(0.406 ; 0.389)$, number of spikelet per panicle $(0.391 ; 0.400)$, per cent spikelet fertility $(0.574 ; 0.588)$ respectively, whereas panicle length showed non-significant positive association. On the contrary plant height $(-0.175 ;-0.209)$ had significantly negative association with grain yield at both the levels. Other yield components, viz., days to $50 \%$ flowering, days to maturity showed non-significant negative association with grain yield (Tables 3 and 4). In general, the genotypic correlation co-efficient were found to be higher than their respective phenotypic correlation coefficients. 
Table.1 Analysis of variance for yield and its component characters in traditional rice genotypes evaluated under control and Low-moisture stress condition

\begin{tabular}{|c|c|c|c|c|c|c|c|c|c|c|c|}
\hline \multirow[t]{2}{*}{ Source of variation } & \multirow[t]{2}{*}{$\mathrm{df}$} & \multicolumn{2}{|c|}{$\begin{array}{c}\text { Days to } 50 \% \\
\text { flowering }\end{array}$} & \multicolumn{2}{|c|}{ Days to maturity } & \multicolumn{2}{|c|}{ Plant height $(\mathrm{cm})$} & \multicolumn{2}{|c|}{ Panicle length $(\mathrm{cm})$} & \multicolumn{2}{|c|}{ Test weight } \\
\hline & & $\mathrm{C}$ & $\mathrm{S}$ & $\mathrm{C}$ & $\mathrm{S}$ & $\mathrm{C}$ & $\mathrm{S}$ & $\mathrm{C}$ & $\mathrm{S}$ & $\mathrm{C}$ & $\mathrm{S}$ \\
\hline Replication & 1 & 0.03 & 31.00 & 1.53 & 116.28 & 82.74 & 63.18 & 23.49 & 29.85 & 0.007 & 13.78 \\
\hline Genotypes (unadjusted) & 63 & 97.98** & 86.08** & 213.16** & $104.93 * *$ & $314.61 * *$ & $161.05 * *$ & $12.25 * *$ & $8.32 * *$ & $26.04 * *$ & $23.57 * *$ \\
\hline Blocks within adjusted & 14 & 4.66 & 8.30 & 12.04 & 8.41 & 9.74 & 7.39 & 3.32 & 2.21 & 0.91 & 0.95 \\
\hline Error intra block & 49 & 7.97 & 6.31 & 7.91 & 7.07 & 6.00 & 5.94 & 2.21 & 1.31 & 1.93 & 1.28 \\
\hline $\mathrm{CD}(5 \%)$ & & 5.20 & 5.18 & 5.86 & 5.44 & 5.13 & 5.00 & 3.16 & 2.40 & 2.42 & 2.18 \\
\hline $\mathrm{CV}(\%)$ & & 2.60 & 2.86 & 2.05 & 2.099 & 3.85 & 4.62 & 10.00 & 8.65 & 6.016 & 5.85 \\
\hline
\end{tabular}

\begin{tabular}{|c|c|c|c|c|c|c|c|c|c|c|c|}
\hline \multirow[t]{2}{*}{ Source of variation } & \multirow[t]{2}{*}{ df } & \multicolumn{2}{|c|}{ No. of Tiller/plant } & \multicolumn{2}{|c|}{$\begin{array}{c}\text { No. of Productive } \\
\text { Tiller/plant }\end{array}$} & \multicolumn{2}{|c|}{$\begin{array}{l}\text { No. of spikelet per } \\
\text { panicle }\end{array}$} & \multicolumn{2}{|c|}{$\begin{array}{c}\text { Per cent of spikelet } \\
\text { fertility }\end{array}$} & \multicolumn{2}{|c|}{ Grain yield $(\mathrm{g}) /$ plant } \\
\hline & & $\mathrm{C}$ & $\mathrm{S}$ & $\mathrm{C}$ & $\mathrm{S}$ & $\mathrm{C}$ & $\mathrm{S}$ & $\mathrm{C}$ & $\mathrm{S}$ & $\mathrm{C}$ & $\mathrm{S}$ \\
\hline Replication & 1 & 29.24 & 53.24 & 36.99 & 10.90 & 0.15 & 7.94 & 30.88 & 1.56 & 39.32 & 5.89 \\
\hline Genotypes (unadjusted) & 63 & $28.63 * *$ & $52.26 * *$ & $20.18 *$ & $22.59 * *$ & $707.27 * *$ & $463.63 * *$ & $122.35 * *$ & $331.69 * *$ & $39.96 * *$ & $26.12 * *$ \\
\hline Blocks within adjusted & 14 & 2.74 & 2.00 & 1.55 & 1.54 & 12.41 & 7.15 & 2.62 & 6.50 & 2.76 & 1.00 \\
\hline Error intra block & 49 & 3.42 & 1.98 & 2.52 & 1.59 & 18.60 & 9.44 & 15.00 & 7.38 & 2.92 & 1.28 \\
\hline $\mathrm{CD}(5 \%)$ & & 3.61 & 2.83 & 2.96 & 2.52 & 8.17 & 5.95 & 8.17 & 5.37 & 3.41 & 2.20 \\
\hline $\mathrm{CV}(\%)$ & & 10.43 & 8.62 & 11.78 & 10.89 & 4.55 & 3.53 & 5.07 & 5.45 & 11.70 & 12.23 \\
\hline
\end{tabular}

C- Control;

S- moisture Stress

Table.2 Estimates of range, mean, variability, heritability and genetic advance for yield and its component characters in traditional Rice genotypes evaluated under control and low-moisture stress condition

\begin{tabular}{|c|c|c|c|c|c|c|c|c|c|c|c|c|c|}
\hline & \multirow[t]{2}{*}{ CHARACTERS } & \multicolumn{2}{|c|}{ MEAN \pm SE } & \multicolumn{2}{|c|}{ RANGE } & \multicolumn{2}{|c|}{$\operatorname{PCV}(\%)$} & \multicolumn{2}{|c|}{ GCV $(\%)$} & \multicolumn{2}{|c|}{$\begin{array}{c}h^{2} \text { broad } \\
\text { sense }(\%)\end{array}$} & \multicolumn{2}{|c|}{ GAM (\%) } \\
\hline & & $\mathbf{C}$ & $\mathbf{S}$ & $\mathbf{C}$ & $\mathbf{S}$ & $\mathbf{C}$ & $\mathbf{S}$ & $\mathbf{C}$ & $\mathbf{S}$ & $\mathbf{C}$ & $\mathbf{S}$ & $\mathbf{C}$ & $\mathbf{S}$ \\
\hline $\mathbf{1}$ & Days to $50 \%$ flowering & $99.59 \pm 2.59$ & $90.16 \pm 2.57$ & $80.50-115.50$ & $75.50-105.00$ & 9.93 & 7.55 & 9.52 & 6.98 & 91.86 & 85.45 & 18.44 & 13.30 \\
\hline 2 & Days to maturity & $141.93 \pm 2.91$ & $128.96 \pm 2.7$ & $116.5-169.5$ & $110.00-149.00$ & 7.42 & 5.81 & 7.12 & 5.41 & 92.04 & 86.87 & 14.07 & 10.39 \\
\hline 3 & Plant height (cm) & $66.21 \pm 2.55$ & $53.82 \pm 2.49$ & $39.75-97.33$ & $30.75-73.00$ & 19.14 & 16.99 & 18.73 & 16.34 & 95.75 & 92.51 & 37.76 & 32.38 \\
\hline 4 & Panicle length $(\mathrm{cm})$ & $15.42 \pm 1.54$ & $13.83 \pm 1.19$ & $11.83-20.66$ & $9.25-20.08$ & 17.58 & 16.03 & 14.34 & 13.33 & 66.52 & 69.21 & 24.09 & 22.86 \\
\hline 5 & Test weight $(\mathrm{g})$ & $20.07 \pm 1.20$ & $18.57 \pm 1.08$ & $13.00-36.00$ & $12.00-34.50$ & 18.55 & 18.94 & 17.38 & 17.99 & 87.70 & 90.24 & 33.53 & 35.22 \\
\hline 6 & No. of tillers per plant & $17.23 \pm 1.79$ & $16.33 \pm 1.40$ & $7.33-29.00$ & $4.66-28.25$ & 23.17 & 31.88 & 20.66 & 30.69 & 79.50 & 92.68 & 37.95 & 60.87 \\
\hline 7 & No. of Productive tillers per plant & $12.52 \pm 1.47$ & $11.54 \pm 1.25$ & $5.50-23.25$ & $5.08-20.25$ & 26.78 & 30.12 & 23.87 & 28.08 & 79.43 & 86.92 & 43.82 & 53.93 \\
\hline 8 & No. of spikelet per panicle & $89.33 \pm 4.06$ & $83.91 \pm 2.96$ & $57.08-150.58$ & $46.83-121.16$ & 21.30 & 18.31 & 20.79 & 17.96 & 95.24 & 96.22 & 41.80 & 36.30 \\
\hline 9 & Per cent of spikelet fertility & $76.31 \pm 4.06$ & $49.02 \pm 2.67$ & $60.94-93.84$ & $29.26-80.52$ & 10.74 & 26.55 & 9.721 & 25.98 & 81.79 & 95.76 & 18.11 & 52.38 \\
\hline $\mathbf{1 0}$ & Grain yield (g)/plant & $14.52 \pm 1.70$ & $8.93 \pm 1.09$ & $7.96-30.60$ & $2.85-22.32$ & 31.87 & 41.18 & 29.64 & 39.29 & 86.50 & 91.04 & 56.78 & 77.23 \\
\hline
\end{tabular}


Table.3 Estimates of phenotypic correlation for yield and root related traits under control and low-moisture stress condition

\begin{tabular}{|c|c|c|c|c|c|c|c|c|c|c|c|c|c|c|}
\hline Traits & $\mathrm{X} 1$ & $\mathrm{X} 2$ & $\mathrm{X} 3$ & $\mathrm{X} 4$ & X5 & X6 & $\mathrm{X} 7$ & X8 & X9 & X10 & X11 & $\mathrm{X} 12$ & $\mathrm{X} 13$ & X14 \\
\hline $\mathrm{X} 1$ & 1 & $0.543 * *$ & $0.341 * *$ & 0.157 & $-0.194 *$ & $0.274 * *$ & $0.338 * *$ & $0.296 * *$ & $0.202 *$ & -0.079 & $-0.180 *$ & $-0.232 * *$ & -0.070 & $0.255^{* *}$ \\
\hline $\mathrm{X} 2$ & $0.601 * *$ & 1 & $0.283 * *$ & -0.010 & -0.170 & $0.324 * *$ & $0.461 * *$ & $0.363 * *$ & $0.185^{*}$ & 0.083 & $-0.250 * *$ & $-0.222 *$ & -0.162 & $0.334 * *$ \\
\hline X3 & -0.042 & $-0.185^{*}$ & 1 & $0.451 * *$ & -0.041 & $0.312 * *$ & 0.168 & -0.008 & 0.042 & 0.055 & -0.084 & 0.006 & -0.080 & 0.079 \\
\hline $\mathrm{X} 4$ & -0.025 & $-0.271 * *$ & $0.448 * *$ & 1 & 0.112 & -0.074 & -0.144 & 0.073 & $0.263 * *$ & 0.122 & -0.029 & $0.175^{*}$ & -0.142 & 0.138 \\
\hline X5 & -0.047 & -0.154 & 0.004 & 0.106 & 1 & -0.056 & -0.086 & $-0.298 * *$ & -0.084 & $0.183^{*}$ & 0.062 & 0.152 & 0.073 & $0.399 * *$ \\
\hline X6 & -0.026 & 0.109 & $-0.253 * *$ & -0.087 & -0.101 & 1 & $0.793 * *$ & 0.004 & 0.113 & 0.015 & -0.056 & -0.082 & 0.064 & $0.359 * *$ \\
\hline $\mathrm{X7}$ & -0.079 & 0.088 & $-0.228 * *$ & -0.079 & -0.115 & $0.807 * *$ & 1 & $0.219^{*}$ & $0.182 *$ & 0.121 & -0.131 & -0.167 & 0.047 & $0.515^{* *}$ \\
\hline $\mathrm{X} 8$ & 0.116 & $0.310 * *$ & -0.133 & 0.114 & $-0.280 * *$ & 0.013 & 0.044 & 1 & 0.153 & -0.005 & -0.142 & $-0.197 *$ & -0.153 & $0.394 * *$ \\
\hline $\mathrm{X} 9$ & $0.173^{*}$ & -0.060 & -0.044 & 0.147 & 0.036 & -0.087 & -0.068 & 0.156 & 1 & 0.107 & -0.142 & -0.008 & -0.155 & $0.441 * *$ \\
\hline $\mathrm{X} 10$ & -0.066 & -0.063 & -0.137 & -0.137 & 0.149 & 0.163 & -0.021 & -0.005 & 0.085 & 1 & 0.094 & $0.322 * *$ & $0.333 * *$ & 0.137 \\
\hline X11 & $-0.262 * *$ & $-0.287 * *$ & 0.015 & -0.006 & 0.091 & -0.044 & -0.055 & -0.025 & -0.015 & 0.107 & 1 & $0.643 * *$ & $0.588 * *$ & -0.135 \\
\hline $\mathrm{X} 12$ & -0.119 & -0.068 & 0.004 & -0.012 & 0.087 & -0.046 & -0.074 & -0.096 & 0.008 & $0.273 * *$ & $0.631 * *$ & 1 & $0.575^{* *}$ & -0.106 \\
\hline $\mathrm{X} 13$ & $-0.205^{*}$ & -0.080 & -0.052 & -0.127 & 0.082 & 0.064 & 0.058 & -0.052 & 0.026 & $0.308 * *$ & $0.466 * *$ & $0.598 * *$ & 1 & -0.087 \\
\hline X14 & -0.060 & -0.029 & $-0.175^{*}$ & 0.147 & $0.250 * *$ & $0.377 * *$ & $0.406 * *$ & $0.391 * *$ & $0.574 * *$ & 0.144 & 0.066 & 0.016 & 0.045 & 1 \\
\hline
\end{tabular}

**Significance at $1 \%, *$ Significance at $5 \%$ (Above diagonal control; below diagonal low-moisture stress).

Where,

$\mathrm{X}_{1}$ - Days to 50 per cent flowering

$\mathrm{X}_{2}$ - Days to maturity

$\mathrm{X}_{3}$ - Plant height

$\mathrm{X}_{4}$ - Panicle length $(\mathrm{cm})$

$\mathrm{X}_{5}$-1000-grain weight (g)
$\mathrm{X}_{6}$ - No. of tiller per plant

$\mathrm{X}_{7}$ - No. of productive tiller per plant

$\mathrm{X}_{8}$ - No. of spikelet per panicle

$\mathrm{X}_{9^{-}}$Per cent of spikelet fertility

$\mathrm{X}_{10}$ - Root length (cm)
$\mathrm{X}_{11^{-}}$Root number

$\mathrm{X}_{12}$ - Root volume(cc)

$\mathrm{X}_{13}$ - Dry root weight $(\mathrm{g})$

$\mathrm{X}_{14-}$ Grain yield $(\mathrm{g})$ per plant 
Table.4 Estimates of genotypic correlation for grain yield and root related traits under control and low-moisture stress condition

\begin{tabular}{|c|c|c|c|c|c|c|c|c|c|c|c|c|c|c|}
\hline Traits & $\mathrm{X} 1$ & $\mathrm{X} 2$ & $\mathrm{X} 3$ & $\mathrm{X} 4$ & $\mathrm{X} 5$ & X6 & $\mathrm{X} 7$ & $\mathrm{X} 8$ & X9 & $\mathrm{X} 10$ & $\mathrm{X} 11$ & $\mathrm{X} 12$ & $\mathrm{X} 13$ & $\mathrm{X} 14$ \\
\hline $\mathrm{X} 1$ & 1 & $0.561 * *$ & $0.358^{* *}$ & $0.213^{*}$ & $-0.221^{*}$ & $0.317^{* *}$ & $0.407 * *$ & $0.318 * *$ & $0.239 * *$ & -0.085 & $-0.199 *$ & $-0.258^{* *}$ & -0.093 & $0.281 * *$ \\
\hline $\mathrm{X} 2$ & $0.643 * *$ & 1 & $0.295^{* *}$ & 0.009 & -0.172 & $0.365^{* *}$ & $0.539 * *$ & $0.402 * *$ & $0.226^{* *}$ & 0.085 & $-0.260 * *$ & $-0.236 * *$ & $-0.177^{*}$ & $0.384 * *$ \\
\hline $\mathrm{X} 3$ & -0.035 & $-0.181^{*}$ & 1 & $0.539 * *$ & -0.039 & $0.330^{* * *}$ & 0.163 & -0.017 & 0.035 & 0.053 & -0.097 & 0.001 & -0.109 & 0.045 \\
\hline $\mathrm{X} 4$ & 0.005 & $-0.317 * *$ & $0.499 * *$ & 1 & $0.186^{*}$ & $-0.178^{*}$ & $-0.392 * *$ & 0.007 & $0.256^{* *}$ & 0.136 & -0.059 & $0.227 * *$ & $-0.205^{*}$ & 0.082 \\
\hline $\mathrm{X} 5$ & -0.047 & -0.123 & -0.015 & 0.116 & 1 & -0.047 & -0.088 & $-0.317 * *$ & -0.089 & $0.209^{*}$ & 0.068 & 0.166 & 0.082 & $0.470 * *$ \\
\hline X6 & 0.009 & 0.165 & $-0.314 * *$ & $-0.226^{*}$ & -0.149 & 1 & $0.859 * *$ & -0.018 & 0.116 & 0.011 & -0.081 & -0.098 & 0.069 & $0.343 * *$ \\
\hline $\mathrm{X} 7$ & -0.064 & 0.150 & $-0.280 * *$ & -0.139 & -0.161 & $0.844 * *$ & 1 & $0.230 * *$ & $0.186^{*}$ & 0.131 & $-0.175^{*}$ & $-0.198^{*}$ & 0.027 & $0.536 * *$ \\
\hline $\mathrm{X} 8$ & 0.131 & $0.330^{* *}$ & -0.136 & 0.110 & $-0.300 * *$ & 0.017 & 0.045 & 1 & 0.138 & -0.001 & -0.141 & $-0.199^{*}$ & -0.154 & $0.403 * *$ \\
\hline X9 & $0.198^{*}$ & -0.056 & -0.055 & $0.203^{*}$ & 0.025 & -0.093 & -0.078 & 0.163 & 1 & 0.102 & -0.167 & -0.011 & $-0.182 *$ & $0.432 * *$ \\
\hline $\mathrm{X} 10$ & -0.081 & -0.080 & -0.142 & -0.167 & 0.171 & $0.178^{*}$ & -0.020 & -0.002 & 0.105 & 1 & 0.089 & $0.324 * *$ & $0.337 * *$ & 0.141 \\
\hline X11 & $-0.273 * *$ & $-0.306 * *$ & 0.016 & 0.001 & 0.091 & -0.048 & -0.065 & -0.023 & -0.011 & 0.106 & 1 & $0.646^{* *}$ & $0.597 * *$ & -0.169 \\
\hline $\mathrm{X} 12$ & -0.129 & -0.067 & 0.001 & -0.036 & 0.091 & -0.051 & -0.081 & -0.097 & 0.012 & $0.271 * *$ & $0.643 * *$ & 1 & $0.589 * *$ & -0.123 \\
\hline $\mathrm{X} 13$ & $-0.220 * *$ & -0.093 & -0.047 & $-0.218^{*}$ & 0.090 & 0.048 & 0.050 & -0.062 & 0.049 & $0.317 * *$ & $0.487 * *$ & $0.635^{* *}$ & 1 & -0.131 \\
\hline $\mathrm{X} 14$ & -0.053 & -0.016 & $-0.209^{*}$ & 0.136 & $0.261 * *$ & $0.371^{* *}$ & $0.389^{* *}$ & $0.400 * *$ & $0.588 * *$ & 0.163 & 0.073 & 0.014 & 0.054 & 1 \\
\hline
\end{tabular}

** Significance at $1 \%, *$ Significance at $5 \%$ (above diagonal control; below diagonal low-moisture stress).

Where,

$\mathrm{X}_{1}$ - Days to 50 per cent flowering

$\mathrm{X}_{2}$ - Days to maturity

$\mathrm{X}_{3}$ - Plant height

$\mathrm{X}_{4}$ - Panicle Length $(\mathrm{cm})$

$\mathrm{X}_{5}-1000$-grain weight $(\mathrm{g})$
$\mathrm{X}_{6}$ - No. of tiller per plant

$\mathrm{X}_{7}$ - No. of productive tiller per plant

$\mathrm{X}_{8}$ - No. of spikelet per panicle

$\mathrm{X}_{9^{-}}$Per cent of spikelet fertility

$\mathrm{X}_{10}$ - Root length $(\mathrm{cm})$
$\mathrm{X}_{11}$ - Root number

$\mathrm{X}_{12}$ - Root volume (cc)

$\mathrm{X}_{13}$ - Dry root weight (g)

$\mathrm{X}_{14-}$ Grain yield $(\mathrm{g})$ per plant 
Table.5 Estimates of direct and indirect effects of yield components on grain yield at phenotypic level under control condition

\begin{tabular}{|c|c|c|c|c|c|c|c|c|c|c|c|c|c|}
\hline Traits & $\mathrm{X} 1$ & $\mathrm{X} 2$ & X3 & $\mathrm{X} 4$ & X5 & X6 & X7 & X8 & X9 & X10 & X11 & X12 & X13 \\
\hline $\mathrm{X} 1$ & 0.014 & 0.007 & 0.004 & 0.002 & -0.002 & 0.003 & 0.004 & 0.004 & 0.002 & -0.001 & -0.002 & -0.003 & -0.001 \\
\hline $\mathrm{X} 2$ & 0.018 & 0.033 & 0.009 & -0.004 & -0.005 & 0.010 & 0.015 & 0.012 & 0.006 & 0.002 & -0.008 & -0.007 & -0.005 \\
\hline X3 & 0.005 & 0.004 & 0.001 & 0.006 & -0.001 & 0.004 & 0.002 & 0.000 & 0.001 & 0.001 & -0.001 & 0.000 & -0.001 \\
\hline $\mathrm{X} 4$ & 0.001 & -0.001 & 0.004 & 0.010 & 0.001 & -0.008 & -0.001 & 0.007 & 0.002 & 0.001 & -0.003 & 0.002 & -0.001 \\
\hline $\mathrm{X} 5$ & -0.118 & -0.104 & -0.025 & 0.068 & 0.610 & -0.034 & -0.053 & -0.181 & -0.051 & 0.112 & 0.038 & 0.092 & 0.044 \\
\hline X6 & 0.018 & 0.021 & 0.021 & -0.005 & -0.003 & 0.067 & 0.053 & 0.003 & 0.007 & 0.001 & -0.003 & -0.005 & 0.004 \\
\hline X7 & 0.114 & 0.156 & 0.057 & -0.048 & -0.029 & 0.269 & 0.339 & 0.074 & 0.602 & 0.041 & -0.044 & -0.056 & 0.016 \\
\hline X8 & 0.126 & 0.154 & -0.003 & 0.031 & -0.126 & 0.001 & 0.093 & 0.425 & 0.065 & -0.002 & -0.060 & -0.084 & -0.065 \\
\hline X9 & 0.072 & 0.065 & 0.015 & 0.093 & -0.029 & 0.040 & 0.064 & 0.054 & 0.354 & 0.038 & -0.050 & -0.002 & -0.055 \\
\hline X10 & 0.003 & -0.003 & -0.002 & -0.005 & -0.008 & -0.007 & -0.005 & 0.000 & -0.004 & -0.045 & -0.004 & -0.014 & -0.015 \\
\hline X11 & -0.005 & -0.008 & -0.002 & -0.001 & 0.002 & -0.001 & -0.004 & -0.004 & -0.004 & 0.003 & 0.031 & 0.020 & 0.018 \\
\hline X12 & 0.010 & 0.010 & -0.003 & -0.008 & -0.006 & 0.003 & 0.007 & 0.009 & 0.004 & -0.014 & -0.029 & -0.045 & -0.026 \\
\hline X13 & 0.001 & 0.003 & 0.002 & 0.003 & -0.001 & -0.001 & -0.001 & 0.003 & 0.003 & -0.007 & -0.001 & -0.001 & -0.002 \\
\hline$r$ value & 0.255 & 0.334 & 0.079 & 0.137 & 0.398 & 0.357 & 0.515 & 0.393 & 0.443 & 0.138 & -0.137 & -0.105 & -0.083 \\
\hline
\end{tabular}

Residual effect $=0.471$

Where,

$\mathrm{X}_{1}$ - Days to 50 per cent flowering

$\mathrm{X}_{2}$ - Days to maturity

$\mathrm{X}_{3}$ - Plant height

$\mathrm{X}_{4}$ - Panicle Length $(\mathrm{cm})$

$\mathrm{X}_{5}$-1000-grain weight (g)
$\mathrm{X}_{6}$ - No. of tiller per plant

$\mathrm{X}_{7}$ - No. of productive tiller per plant

$\mathrm{X}_{8}$ - No. of spikelet per panicle

$\mathrm{X}_{9}$ - Per cent of spikelet fertility

$\mathrm{X}_{10}$ - Root length (cm)
$\mathrm{X}_{11}$ - Root number

$\mathrm{X}_{12}$ - Root volume (cc)

$\mathrm{X}_{13}$ - Dry root weight (g) 
Table.6 Estimates of direct and indirect effects of yield components on grain yield at phenotypic level under low-moisture stress condition

\begin{tabular}{|c|c|c|c|c|c|c|c|c|c|c|c|c|c|}
\hline Traits & $\mathrm{X} 1$ & $\mathrm{X} 2$ & X3 & $\mathrm{X} 4$ & X5 & X6 & $\mathrm{X} 7$ & X8 & X9 & X10 & X11 & X12 & X13 \\
\hline $\mathrm{X} 1$ & -0.150 & -0.090 & 0.006 & 0.003 & 0.007 & 0.004 & 0.012 & -0.017 & -0.026 & 0.010 & 0.039 & 0.018 & 0.031 \\
\hline $\mathrm{X} 2$ & -0.014 & -0.023 & 0.004 & 0.006 & 0.003 & -0.002 & -0.002 & -0.007 & 0.001 & 0.001 & 0.006 & 0.001 & 0.001 \\
\hline X3 & -0.001 & -0.004 & 0.022 & 0.010 & 0.001 & -0.005 & -0.005 & -0.003 & -0.001 & -0.003 & 0.004 & 0.001 & -0.001 \\
\hline $\mathrm{X} 4$ & 0.004 & 0.004 & -0.007 & -0.010 & -0.001 & 0.001 & 0.001 & -0.001 & -0.002 & 0.002 & 0.001 & 0.002 & 0.002 \\
\hline X5 & -0.018 & -0.061 & 0.001 & 0.042 & 0.398 & -0.040 & -0.046 & -0.116 & 0.014 & 0.059 & 0.036 & 0.034 & 0.033 \\
\hline X6 & -0.006 & 0.028 & -0.065 & -0.022 & -0.025 & 0.256 & 0.206 & 0.003 & -0.022 & 0.041 & -0.011 & -0.011 & 0.016 \\
\hline X7 & -0.021 & 0.024 & -0.062 & -0.021 & -0.031 & 0.219 & 0.272 & 0.012 & -0.018 & -0.005 & -0.015 & -0.020 & 0.016 \\
\hline X8 & 0.050 & 0.134 & -0.057 & 0.049 & -0.121 & 0.005 & 0.019 & 0.432 & 0.067 & -0.002 & -0.011 & -0.041 & -0.022 \\
\hline X9 & 0.097 & -0.034 & -0.025 & 0.082 & 0.020 & -0.049 & -0.038 & 0.088 & 0.563 & 0.047 & -0.008 & 0.004 & 0.015 \\
\hline X10 & -0.001 & -0.001 & -0.002 & -0.002 & 0.002 & 0.003 & 0.000 & 0.000 & 0.001 & 0.001 & 0.002 & 0.004 & 0.005 \\
\hline X11 & -0.008 & -0.009 & 0.005 & -0.002 & 0.003 & -0.001 & -0.001 & -0.008 & -0.005 & 0.003 & 0.032 & 0.020 & 0.015 \\
\hline X12 & -0.008 & -0.004 & 0.003 & -0.009 & 0.006 & -0.003 & -0.005 & -0.007 & 0.006 & 0.019 & 0.045 & 0.072 & 0.043 \\
\hline X13 & 0.021 & 0.008 & 0.005 & 0.013 & -0.008 & -0.006 & -0.006 & 0.005 & -0.002 & -0.032 & -0.048 & -0.062 & -0.104 \\
\hline r value & -0.060 & -0.028 & -0.175 & 0.148 & 0.248 & 0.375 & 0.402 & 0.391 & 0.574 & 0.144 & 0.067 & 0.016 & 0.045 \\
\hline
\end{tabular}

Residual effect $=0.444$

Where,

$\mathrm{X}_{1}$ - Days to 50 per cent flowering

$\mathrm{X}_{2}$ - Days to maturity

$\mathrm{X}_{3}$ - Plant height

$\mathrm{X}_{4}$ - Panicle Length $(\mathrm{cm})$

$\mathrm{X}_{5}-1000$-grain weight (g)
$\mathrm{X}_{6}$ - No. of tiller per plant

$\mathrm{X}_{7}$ - No. of productive tiller per plant

$\mathrm{X}_{8}$ - No. of spikelet per panicle

$\mathrm{X}_{9}$ - Per cent of spikelet fertility

$\mathrm{X}_{10}$ - Root length $(\mathrm{cm})$
$\mathrm{X}_{11}$ - Root number

$\mathrm{X}_{12}$ - Root volume (cc)

$\mathrm{X}_{13}$ - Dry root weight (g) 
Appendix.1 List of 64 traditional rice genotypes are taken for investigation

\begin{tabular}{|c|c|c|c|}
\hline Sl. No & GENOTYES & Sl. No & GENOTYES \\
\hline 1 & DODDAMULLARE & 33 & ANDRABASUMATHI \\
\hline 2 & CHINA-988* & 34 & HMT \\
\hline 3 & SKAU-98* & 35 & KOUGISAALE \\
\hline 4 & K-336* & 36 & CHINANAPUNNI \\
\hline 5 & SKAU-23* & 37 & $\mathrm{BI}-33 *$ \\
\hline 6 & MGD-101* & 38 & NMS-2* \\
\hline 7 & $\mathrm{CH}-1007 *$ & 39 & BADASHIPARIMALAKKI \\
\hline 8 & WAZULKREER & 40 & MATTAKHARA \\
\hline 9 & JAYA199* & 41 & ABHILASHA \\
\hline 10 & SRI-214* & 42 & NAVALAISAALE \\
\hline 11 & KYASARI-202* & 43 & MUKKANNA \\
\hline 12 & UMA-213* & 44 & ATHNALU \\
\hline 13 & SALAMSANNA & 45 & MYSORE MALLIGE \\
\hline 14 & SK339* & 46 & BANGARADAGUNDU \\
\hline 15 & ZADAGI & 47 & BANGLARICE \\
\hline 16 & SKAU-334* & 48 & ELATAGYAGIDDA \\
\hline 17 & CHAMPAKALI & 49 & BANGARU SANNA \\
\hline 18 & $\mathrm{~J}-192 *$ & 50 & JEERISANNA \\
\hline 19 & BANAVASISELECTION & 51 & PADMAREKHA \\
\hline 20 & ASHOKA-228F* & 52 & KUSHIADIKSHAM \\
\hline 21 & JEERGA & 53 & IR-64* \\
\hline 22 & ANEKOMBINABATTA & 54 & AZUCENA \\
\hline 23 & HUGGIBHATTA & 55 & MALLIGE \\
\hline 24 & SALAMBATTIBHARA & 56 & KICHADISAAMBA \\
\hline 25 & DAPPA BATTA & 57 & BASUMATHI \\
\hline 26 & KH-10/NMS-2* & 58 & BURMABLACK \\
\hline 27 & KAGGA & 59 & DELHIBASUMATHI \\
\hline 28 & KUDUVEKALANJI & 60 & DODDIGA \\
\hline 29 & GOWRISANNA & 61 & KADUVALAI \\
\hline 30 & MADILAISAAMBA & 62 & KARIMUNDAGA \\
\hline 31 & INTIN & 63 & SAMPIGE \\
\hline 32 & KEMPUKHARU & 64 & DODDIBHATTA \\
\hline
\end{tabular}

* Improved lines/varieties used as checks 
This was in conformity with the findings of Shashidhar et al., (2005) and Muthuramu et $a l$. , (2010). Hence, indirect selection for grain yield could be effectively done through direct selection of yield component characters as indicated above.

\section{Direct and indirect effects}

Ten out of 14 characters had positive and direct effect on grain yield at phenotypic level under control condition. The characters which had positive direct effects are days to 50 percent flowering (0.014), days to maturity (0.033), plant height $(0.001)$, panicle length (0.010), 1000-grain weight (0.610), number of tillers per plant (0.067), number of productive tillers per plant (0.339), number of spikelet per panicle $(0.425)$, per cent spikelet fertility (0.354) and root number (0.031). Other characters viz., root length $(-0.045)$, root volume (-0.045) and dry root weight (-0.002) had negative direct effects on grain yield (Table 5). Nine out of 14 characters had positive and direct effect on grain yield at phenotypic level under low-moisture stress condition. The characters which had positive direct effects are plant height (0.022), 1000grain weight (0.398), number of tillers per plant (0.256), number of productive tillers per plant (0.272), number of spikelet per panicle (0.432), per cent spikelet fertility (0.563), This result agrees with the research findings of Yogameenakshi et al., (2004) and Panwar et al., (2007). Other characters viz., days to 50 per cent flowering (-0.150), days to maturity $(-0.023)$, panicle length $(-0.010)$ and dry root weight $(-0.104)$ had negative direct effects on grain yield (Table 6). 1000-grain weight (0.610) in control and per cent spikelet fertility (0.563) in low-moisture stress condition had the highest positive direct effect, the forgone discussion on path coefficient analysis has clearly demonstrated that due weightage to days to 50 per cent flowering, plant height, test weight, number of productive tiller per plant, per cent spikelet fertility, are helpful in increasing the grain yield in rice through selection under lowmoisture stress conditions. The residual effect (0.47 in control and 0.44 in low-moisture stress) was low, indicating that much of the variation in yield has been accounted by the characters studied and that the choice of characters was appropriate.

\section{References}

Abarshahr, M., Rabiei, B. and Lahigi, H. S., 2011, Genetic variability, correlation and path analysis in rice under optimum and stress irrigation regimes. Nat. Sci. Biol., 3(4):134-142.

Cochran, W. G. and Cox, G. M., 1957, Experimental Designs. John Wiley and Sons, Inc., New York, 611.

Das, S., Subudhi, H. N. and Reddy, J. N., 2001, Genetic variability in Grain quality characteristics and yield in lowland rice genotypes. Oryza, 44(4): 343-346.

Dewey, D and Lu, K.H. 1959. A correlation and path coefficient analysis of components of crested wheat grass seed production. Agron. J. 51:515-518.

Falconer, D. S., 1981, Introduction to Quantitative Genetics, 2nd edition, Longman Group Limited, Longman Burnt Mills, Harlow, Essex, England.'

Hanson, C. H., Robinson, H. F. and Comstock, R. E., 1956, Biometrical studies of yield in segregating population of Korea Lespedeza. Agron. J., 48: 267- 282.

Johannsen, W. L., 1909, Elementadorexakten Erbik. Int. Jena: Gustav Tisher. pp: 515.

Johnson, H. W., Robinson, H. F. and Comstock, R. E., 1955, Estimates of genetic and environmental variability in soybean. Agron. J., 7(7): 314-318.

Kuldeep, T., Bathshwar, K., Ramesh, B., and Ajay, T., 2004, Genetic variability and 
correlations for some seedlings and mature plant traits in 70 genotypes of rice. Res. Crops, 5(1): 60-65.

Lush, J. L., 1945, Intra- sire correlation on regression of off- spring on dams as a method of estimating heritability of characters. Proc. Am. Soc. Animal Prod., 33: 292-301.

Madhusudhan, 2001, Development of trait differential lines and detection of near isogenic lines by phenotypic and molecular characterization in early segregating generation in rice (Oryza sativa L.). M. Sc. (Agri.) Thesis, Univ. Agric. Sci., Bangalore. pp: 96.

Muthuramu, S., Jebaraj, S. and Gnanasekaran, M., 2010, Association analysis for drought tolerance in rice (Oryza sativa L.). Res J. Agri Sci., 1(4): 426-42.

Nagabhushan, K., 2003. Detection 0-1 main QTL controlling plant traits and identification of RAPD markers associated with plant height in rice (Oryza sativa L.). M. Sc. (Agri.) Thesis, Univ. Agric. Sci., Bangalore. pp: 136.

Osman, K. A., Mustafa, A. M., Ali, F., Yonglain, Z.and Fazhan, Q., 2012, Genetic variability for yield and related attributes of upland rice genotypes in semi-arid zone (Sudan). Afri. J. Agric., 7(33): 4613-4619.

Pandey, V. R., Singh, P. K., Verma, O. P. and
Pandey. P, 2012, Interrelationship and path coefficient estimation in rice under salt stress environment. Int. J. Agric. Res., 7(4): 169-184.

Panwar, A., Dhaka, R. P. S. and Vinodkumar, 2007, Path analysis of grain yield in rice. Adv. Plant Sci., 20:27-28.

Remabai, N., Regina, A., Devika, R. and Joseph, C. A., 1992, Genetic variability and association of characters in medium duration rice genotypes. Oryza, 29: 1922.

Shashidhar, H. E., Pasha, F., Janmatti, M., Vinod, M. S and Kanbar, A., 2005, Correlation and path coefficient analysis in traditional cultivars and double haploid lines of rainfed lowland rice. Oryza, 42: 156-159.

Snedecor, G.W and Cochran, W.G. 1967. Statistical Methods. The Iowa State College Press, Ames, Iowa. U.S.A. Pp. 160-413.

Standard Evaluation System for Rice. 1996. Prepared by INGER - Genetic Resources Center, International Rice Research Institute, Manila, Philippines. Pp. 1-39.

Yogameenakshi, P., Nadarajan. And Ambumalarmathi, J., 2004, Correlation and path analysis on yield and drought tolerant in rice (Oryza sativa L.) under drought stress. Oryza, 41: 68-70.

\section{How to cite this article:}

Uday Kumar, H.R., S. Gangaprasad, P. Raghavendra and Dushyantha Kumar, B.M. 2017. Revealing Genetic Variability and Trait Association Studies in Landraces of Rice (Oryza sativa L.) Under Controlled and Drought Conditions. Int.J.Curr.Microbiol.App.Sci. 6(8): 737-747. doi: https://doi.org/10.20546/ijcmas.2017.608.094 